\title{
PASSIO. \\ COMPOSICIÓN MUSICAL PARA MARIMBA, DANZA, CAPTACIÓN DE MOVIMIENTO Y ELECTRÓNICA EN VIVO PRODUCIDA EN IRCAM-CENTRE POMPIDOU, PARÍS
}

\author{
Alberto Carretero Aguado \\ Conservatorio Superior de Música «Manuel Castillo» (Sevilla)
}

Fecha de recepción: 18/07/2021

Fecha de aceptación: 08/10/2021

\section{Resumen}

Este proyecto interdisciplinar se llevó a cabo en el IRCAM-Centre Pompidou de París como una colaboración entre dos creadores: la coreógrafa Christine Gérard y el compositor Alberto Carretero; así como dos intérpretes: la bailarina Aurélie Berland y la percusionista Linda Edsjö. Se trata de un proceso basado en la escritura coreográfica y musical conjunta, tomando como inspiración la notación Laban para danza. A partir del concepto de «pasión», se buscaron materiales sonoros y gestuales que fueran apropiados para representar y dramatizar de forma abstracta este concepto a través de un itinerario temporal y espacial basado en la geometría de un cubo. Se creó un espacio lumínico en el escenario y un espacio sonoro en la sala mediante un sistema envolvente de altavoces, de acuerdo a la arquitectura de la pieza. Además, existe un trabajo interactivo mediante tecnologías de captación y reconocimiento del movimiento a través de una cámara con sensores infrarrojos, capaz de capturar una imagen 3D y realizar un seguimiento en la oscuridad.

El procesamiento electrónico utiliza técnicas de síntesis y transformación del sonido en tiempo real, tales como modelos físicos, Super phase vocoder (SuperVP), modelos de espacialización y reverberación basados en convolución, armonizadores, sampling, filtros y resonadores, granuladores, líneas de retardo de tiempo variable o flanger.

Palabras clave: Composición musical, partitura, música electrónica, interdisciplinariedad, percusión, danza.

\section{PASSIO \\ MUSICAL COMPOSITION FOR MARIMBA, DANCE, MOTION CAPTURE AND LIVE ELECTRONICS PRODUCED AT IRCAM-CENTRE POMPIDOU, PARIS}

\begin{abstract}
This interdisciplinary project was carried out at the IRCAM-Centre Pompidou in Paris as a collaboration between two creators: the choreographer Christine Gérard and the composer Alberto Carretero; as well as two performers: the dancer Aurélie Berland and the percussionist
\end{abstract}


Enclaves. Revista de Literatura, Música y Artes Escénicas, n. ${ }^{\circ}$ 1, 2021, pp. 196-213. e-ISSN 2792-7350

Alberto Carretero Aguado, «PASSIO. Composición musical para marimba, danza, captación de movimiento y electrónica en vivo producida en IRCAM-

Centre Pompidou, París», https://dx.doi.org/10.12795/enclaves.2021.i01.13

Linda Edsjö. It is a process based on joint choreographic and musical writing, inspired by the Laban notation for dance. From the concept of «passion», sound and gestural materials were sought that were appropriate to abstractly represent and dramatise this concept through a temporal and spatial itinerary based on the geometry of a cube. A light space was created on the stage and a sound space in the hall through a surround system of loudspeakers, in accordance with the architecture of the piece. Therefore, there is an interactive work using motion capture and recognition technologies through a camera with infrared sensors, capable of capturing a 3D image and tracking movement in the dark.

The electronic processing uses real-time sound synthesis and transformation techniques, such as physical models, Super phase vocoder (SuperVP), convolution-based spatialisation and reverberation models, harmonisers, sampling, filters and resonators, granulators, variable time delay lines or flanger.

Keywords: Musical composition, Score, Electronic music, Interdisciplinarity, Percussion, Dance.

\section{Sumario}

1. Creación artística o denominación del proyecto artístico..............................197

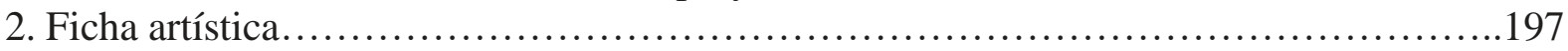

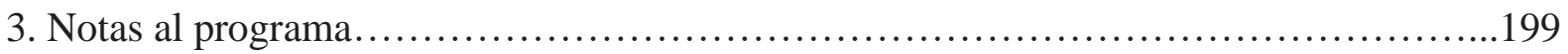

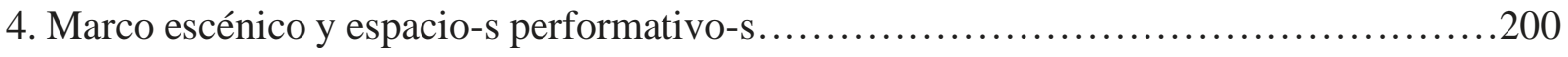

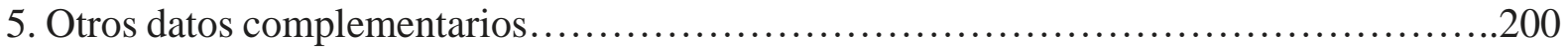

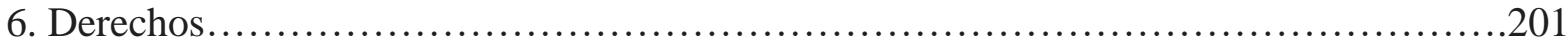

7. Imagen del patch MAX/MSP para el control de la electrónica en vivo...................201

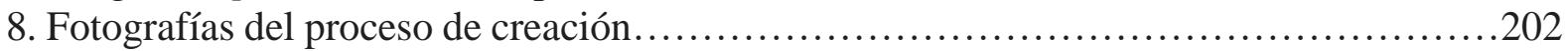

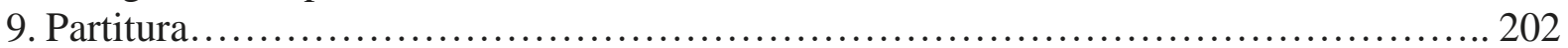

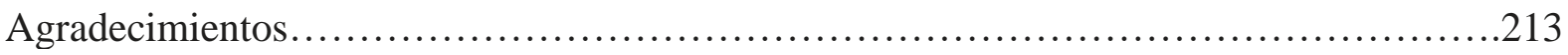

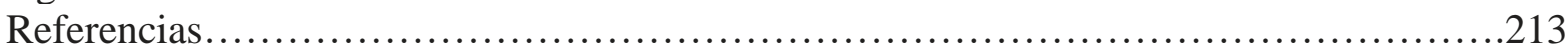

\section{Creación artística o denominación del proyecto artístico}

Título: Passio (2013).

Tipo de creación: Composición musical para marimba, danza, captación de movimiento y electrónica en vivo.

Autoría principal: Alberto CARRETERO (Sevilla, 1985-).

Fecha y lugar de estreno: 30/06/2013. Centre Georges Pompidou, Grande Salle. París (Francia).

\section{Ficha artística}

Producción IRCAM-Centre Pompidou (París, Francia) - Charleroi Danses (Bruselas, Bélgica) Festival Manifeste 2013 - In vivo danse. 
Enclaves. Revista de Literatura, Música y Artes Escénicas, n.o 1, 2021, pp. 196-213. e-ISSN 2792-7350

Alberto Carretero Aguado, «PASSIO. Composición musical para marimba, danza, captación de movimiento y electrónica en vivo producida en IRCAMCentre Pompidou, París», https://dx.doi.org/10.12795/enclaves.2021.i01.13

Percusión (marimba): Linda Edsjö (http://lindaedsjo.com).

Coreografía: Christine Gérard

(http://www.christinegerardchoregraphe.com/p/biographie.html).

Bailarina: Aurélie Berland (http://www.cie-gramma.aurelieberland.com/aurelie-berland/).

Realización: Benoit Meudic (https://www.discogs.com/es/artist/3458841-Benoit-Meudic) (https://www.centrepompidou.fr/es/ressources/personne/UQgl3jn).

Iluminación: Léandre García Lamolla.

Equipo técnico: IRCAM-Centre Pompidou.

Compositor: Alberto Carretero (http://albertocarretero.com).

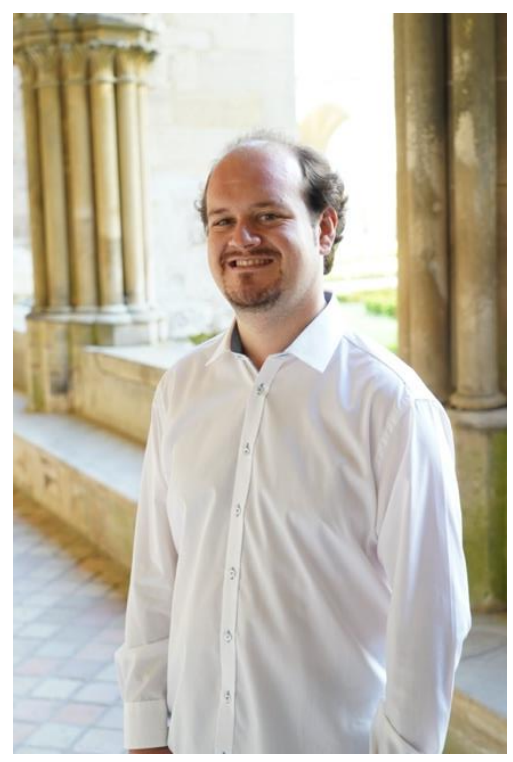

Alberto Carretero (Sevilla, 1985) estudió Composición, con Premio Extraordinario Fin de Carrera, en el Conservatorio Superior de Música «Manuel Castillo» de Sevilla, bajo la tutela de Luis Ignacio Marín, Antonio José Flores y Juan Antonio Pedrosa. Es Ingeniero Superior Informático con Premio Extraordinario, Licenciado en Historia y Ciencias de la Música, Graduado en Periodismo con Premio Extraordinario, Postgraduado en Composición Musical, Máster Oficial en Creación e Interpretación Musical y Doctor en Artes con Sobresaliente Cum Laude por unanimidad y Premio Extraordinario de Doctorado. Ha formado parte del Grupo de Investigación en Topología Computacional en la Universidad de Sevilla y ha dirigido numerosos trabajos de investigación en diversos centros de educación superior.

Entre los premios obtenidos, destacan el Premio Caja Madrid «Andaluces del Futuro», el Premio de Composición de Música Contemporánea «Injuve», el Premio «Real Maestranza» entregado por S. M. El Rey de España, el Segundo Premio de Composición orquestal «Antón García Abril» (Madrid), el Premio «Jóvenes Compositores» de PluralEnsemble, el Premio «Ciudad de Sevilla» del consistorio hispalense, el Premio de Encargo Sinfónico SGAE-AEOS, el Premio «Ensemble Flashback Perpignan», el Accésit del Premio del Consejo Social de la Universidad Rey Juan Carlos, el Premio «Sevilla Joven» de la Consejería de la Presidencia de la Junta de Andalucía; además, disfrutó de la Beca Leonardo de la Fundación BBVA. Ha sido finalista de los premios de Composición «Niccolò Castiglioni» y «San Fedele» (Milán), Premio «Carmelo Bernaola» (Madrid), Premio de Composición Città di Udine y Premio de Composición del Pabellón de Italia (Expo 2015 de Milán).

Se ha especializado en España, Francia, Alemania, Italia, Holanda y Polonia con los maestros Cristóbal Halffter, José María Sánchez-Verdú, Mauricio Sotelo, José Manuel López López, Hèctor Parra, Jesús Rueda, Tomás Marco, Luis de Pablo, Elena Mendoza, Alberto Posadas, César Camarero, Agustín Charles, Brian Ferneyhough, Martín Matalón, Beat Furrer, Kaija Saariaho, Philippe Hurel, Salvatore Sciarrino, Stefano Gervasoni, Helmut Lachenmann, 
Enclaves. Revista de Literatura, Música y Artes Escénicas, n.o 1, 2021, pp. 196-213. e-ISSN 2792-7350

Alberto Carretero Aguado, «PASSIO. Composición musical para marimba, danza, captación de movimiento y electrónica en vivo producida en IRCAMCentre Pompidou, París», https://dx.doi.org/10.12795/enclaves.2021.i01.13

Alessandro Solbiati, Johannes Schöllhorn, Ivan Fedele, Georges Aperghis, Rebecca Saunders, Chaya Czernowin, Mark André, Thierry de Mey, etc.

Su música se ha interpretado en el Carnegie Hall de New York, Centro Pompidou (París), CentQuatre (París), Abadía de Royaumont (Francia), Auditorio Nacional de Música de Madrid, Museo Reina Sofía de Madrid, Ran Baron Hall de Tel Aviv, Auditorio San Fedele de Milán, Teatro Central de Sevilla, Teatro de la Maestranza de Sevilla, Festival San Giovanni Valdarno de Florencia, Sibelius Academy de Helsinki (Tenso Network), Fonderie Kugler de Ginebra, Casa della Musica de Parma, Festival Internacional de Órgano de León, Curso Internacional de Darmstadt, Festival de Música Española (Cádiz), Crículo de Bellas Artes (Madrid), Fundación Juan March (Madrid), Mostra Sonora de Sueca, Teatro Talía de Valencia, Teatro Villamarta de Jerez de la Frontera, etc. Entre sus proyectos se encuentran también colaboraciones con otras artes escénicas y visuales. Ha trabajado con Klangforum Wien, Musikfabrik, Ensemble Recherche, Ensemble Intercontemporain, Real Orquesta Sinfónica de Sevilla, Orquesta SWR de Stuttgart, PluralEnsemble, Ensemble Schallfeld, Meitar Ensemble, Taller Sonoro, Barcelona216, Neopercusión, Cosmos21, Sax-Ensemble, Helsinki Chamber Choir-Tenso, Raquel Andueza, Asier Polo, Manuel Blanco, Juanjo Guillem, Philippe Spiesser, Eva Reiter, Domenico Melchiorre, Pedro Rojas, dissonArt Ensemble, El Perro Andaluz Ensemble, Proyecto Ocnos, Daniel Oyarzábal, Ryoko Aoki, Mario Prisuelos, Grup Instrumental de València, Dhamar, Alberto Rosado, Alfonso Padilla, S'ensemble, Andrés Marín, etc. Sus trabajos han sido grabados por Verso, Tañidos, La Mà de Guido, Columna Música (Revista Sibila patrocinada por la fundación BBVA), Radio Nacional de España y Radio Círculo.

En el campo de las nuevas tecnologías, ha compuesto música electroacústica para la Bienal Internacional de Arte Contemporáneo de Sevilla, la «Noche de los Museos» de Sevilla, la «Noche de los Libros» de Madrid, «In Sonora» de Madrid y «Phonos» de Barcelona. Ha participado en las tres últimas ediciones del Festival Manifeste y en el Congreso Internacional de Efectos Digitales de Audio en el IRCAM (París), así como en el festival Matrix del SWR ExperimentalStudio en Friburgo, Ámsterdam y Varsovia. Ha sido ponente en el Congreso Internacional de Música y Tecnologías de la Universidad de Sevilla y profesor en el Real Conservatorio Superior de Música de Madrid. Es autor de varios libros y artículos de investigación sobre composición, análisis y tecnología musical, así como asiduo profesor invitado en conferencias, congresos, seminarios y clases magistrales. Actualmente es Catedrático de Composición por oposición en el Conservatorio Superior de Música «Manuel Castillo» de Sevilla.

Alberto Carretero, compositor Catedrático de Composición-Departamento de Composición Conservatorio Superior de Música «Manuel Castillo» de Sevilla acarretero@consev.es

https://soundcloud.com/albertocarretero https://www.youtube.com/user/albertocarretero http://albertocarretero.com

\section{Notas al programa}

La palabra «passio» concentra significados muy diversos que aluden a estados límite de la expresión humana, como el amor extremo, el padecimiento, la no-acción o la afición vehemente. La pieza, que nace de un proyecto coreográfico, describe un recorrido por 
Enclaves. Revista de Literatura, Música y Artes Escénicas, n. ${ }^{\circ}$ 1, 2021, pp. 196-213. e-ISSN 2792-7350

Alberto Carretero Aguado, «PASSIO. Composición musical para marimba,

diferentes espacios-pasiones compuestos de materia y gestos musicales basados en los sonidos de madera, metal, vidrio y otros materiales que se pueden extraer mediante la transformación electrónica de la marimba. En esta obra, la gestualidad del instrumentista se convierte en danza que discurre entre estos espacios y pasiones, donde las sonoridades flamencas parecen resonar.

\section{Marco escénico y espacio-s performativo-s}

Esquema del escenario y materiales danza/música/captación de gesto con cámara infrarroja:

KINECT CAM

\begin{tabular}{|c|c|c|}
\hline \multicolumn{2}{|c|}{ Linealidad, equilibrio, líneas, curvas } \\
\hline $\begin{array}{c}\text { Centro-Arriba } \\
\text { Detalles, cuello, manos, cara, garza }\end{array}$ & $\begin{array}{c}\text { Centro-Abajo } \\
\text { Animalidad, primitivismo, arrastrado }\end{array}$ \\
\hline $\begin{array}{c}\text { Frente-Abajo } \\
\text { Pequeños gestos, base } \\
\text { interna, contorsión }\end{array}$ & $\begin{array}{c}\text { Frente-Medio } \\
\text { Dramático, potente, } \\
\text { expresión de cara }\end{array}$ & $\begin{array}{c}\text { Frente-Arriba } \\
\text { Impulsos de energía, } \\
\text { saltos, lucha, flotación }\end{array}$ \\
\hline \multicolumn{2}{|c|}{ PÚBLICO } \\
\hline
\end{tabular}

Fig. 1.

\section{Otros datos complementarios}

Listado de aspectos destacados:

- Proyecto de Danza-Música

- Notación Laban para danza

- Concepto de «Pasión»

- Estados «límite» de la expresión humana

- Amor extremo, sufrimiento, no-acción, vehemencia

- Itinerario a través de diferentes espacios-pasiones compuestos de materia y gestos musicales

- Captación de gesto y reconocimiento

- Kinect camera (imagen 3D y sensores infrarrojos)

- Seguimiento de movimiento incluso en oscuridad

- Espacio / Material / Iluminación (coreografía y música) 
Enclaves. Revista de Literatura, Música y Artes Escénicas, n.o 1, 2021, pp. 196-213. e-ISSN 2792-7350

Alberto Carretero Aguado, «PASSIO. Composición musical para marimba, danza, captación de movimiento y electrónica en vivo producida en IRCAMCentre Pompidou, París», https://dx.doi.org/10.12795/enclaves.2021.i01.13

\section{Listado de procesos electrónicos:}

- Modelos físicos: Modalys (cristal, metal, madera...)

- SuperVP (Superphase vocoder): tiempo/frecuencia

- Espacialización, reverberación: Spat

- Armonizadores

- Sampling

- Filtros, resonadores

- Multidelay

- Granulador

- Flanger

\section{Derechos}

Todos los derechos legales de la música corresponden al compositor.

Registro SGAE: 13.192.488.

Coreografía original de Christine Gérard.

\section{Imagen del patch MAX/MSP para el control de la electrónica en vivo}

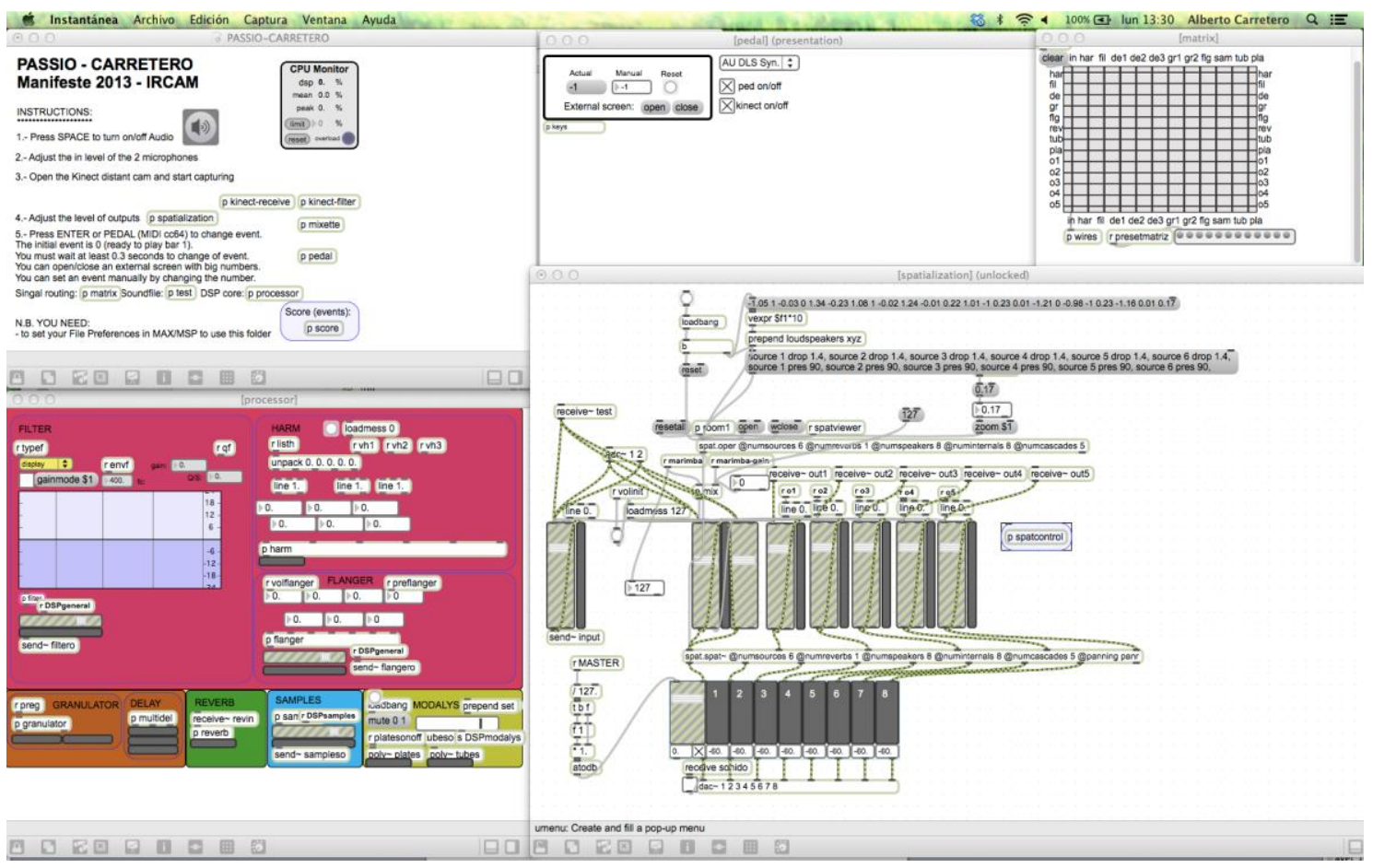

Fig. 2. 
Enclaves. Revista de Literatura, Música y Artes Escénicas, n. ${ }^{\circ}$ 1, 2021, pp. 196-213. e-ISSN 2792-7350

Alberto Carretero Aguado, «PASSIO. Composición musical para marimba, danza, captación de movimiento y electrónica en vivo producida en IRCAMCentre Pompidou, París», https://dx.doi.org/10.12795/enclaves.2021.i01.13

\section{9:nclaves}

Revista de Literatura, Música y Artes Escénicas

\section{Fotografías del proceso de creación}
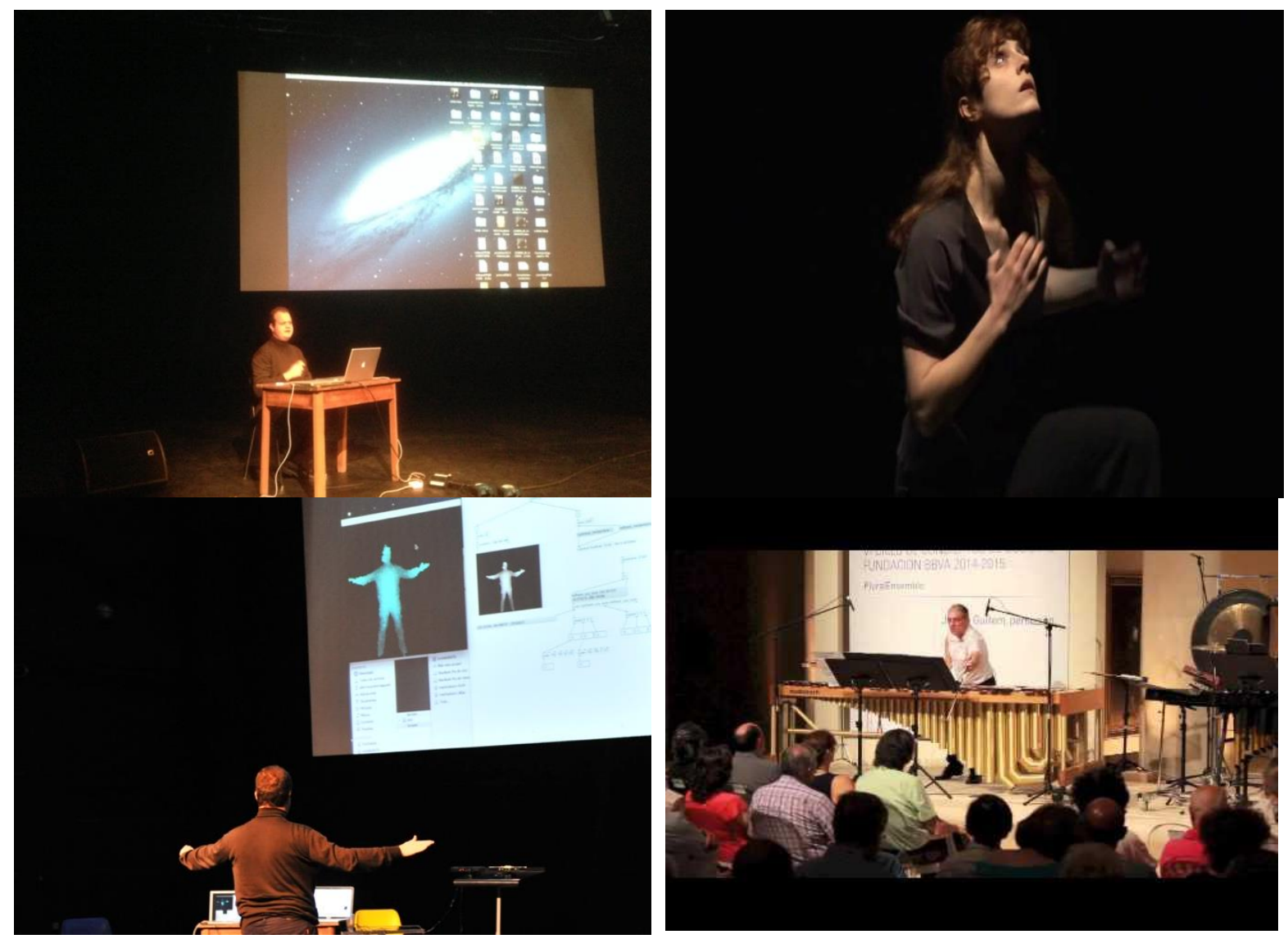

Imágenes 1, 2, 3 y 4.

\section{Partitura}

La partitura ha sido generada con Finale. 
Enclaves. Revista de Literatura, Música y Artes Escénicas, n.o 1, 2021, pp. 196-213. e-ISSN 2792-7350

Alberto Carretero Aguado, «PASSIO. Composición musical para marimba, danza, captación de movimiento y electrónica en vivo producida en IRCAMCentre Pompidou, París», https://dx.doi.org/10.12795/enclaves.2021.i01.13

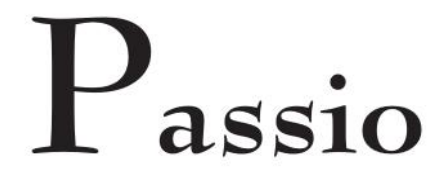

for dancer, marimba and live electronics

Festival MANIFESTE 2013 - IRCAM

\title{
Alberto CARRETERO
}

\author{
Partitura-Score
}

(2013)

Imagen 5. 
Enclaves. Revista de Literatura, Música y Artes Escénicas, n. ${ }^{\circ}$ 1, 2021, pp. 196-213. e-ISSN 2792-7350

Alberto Carretero Aguado, «PASSIO. Composición musical para marimba, danza, captación de movimiento y electrónica en vivo producida en IRCAMCentre Pompidou, París», https://dx.doi.org/10.12795/enclaves.2021.i01.13

\section{$\mathrm{P}_{\text {assio }}$}

\section{PERFORMANCE NOTES}

- Play on the node ( ) or anti-node (ordinary position: $\bigcirc$ ) of the key.

- Thin arrows indicate gradual transitions.

- Thick arrows indicate repetition of a pattern (loop).

- R: rim-shot.

- Alterations affect for whole measures.

- Grace notes must be played before the beat as fast as possible.

- Thin lines crossing notes indicate to play as fast as possible.

- Rasp with 2 "guiro mallets" (reibestab): $z$

- Roll and wipe with 2 superballs: $m$

- Cluster with horizontal mallets: [ (better if one hand plays on "white" keys and the other plays on "black" keys).

- Mallets: hard / medium / soft.

- Harmonics: Touch slightly the center of the key to produce an overtone.

- Glissandi: use a rubber mallet in combination with a bow or another mallet.

- Lighting: some orientations are provided according to the choreography.

- Live electronics: MAX/MSP patch +1 or 2 MIDI pedals +2 or 4 microphones + 8 loudspeakers + kinect camera

- N.B. It is possible to perform this piece as a marimba solo or a marimba + live electronics piece. 
Enclaves. Revista de Literatura, Música y Artes Escénicas, n.o 1, 2021, pp. 196-213. e-ISSN 2792-7350

Alberto Carretero Aguado, «PASSIO. Composición musical para marimba, danza, captación de movimiento y electrónica en vivo producida en IRCAMCentre Pompidou, París», https://dx.doi.org/10.12795/enclaves.2021.i01.13 9:nclaves

Revista de Literatura, Música y Artes Escénicas
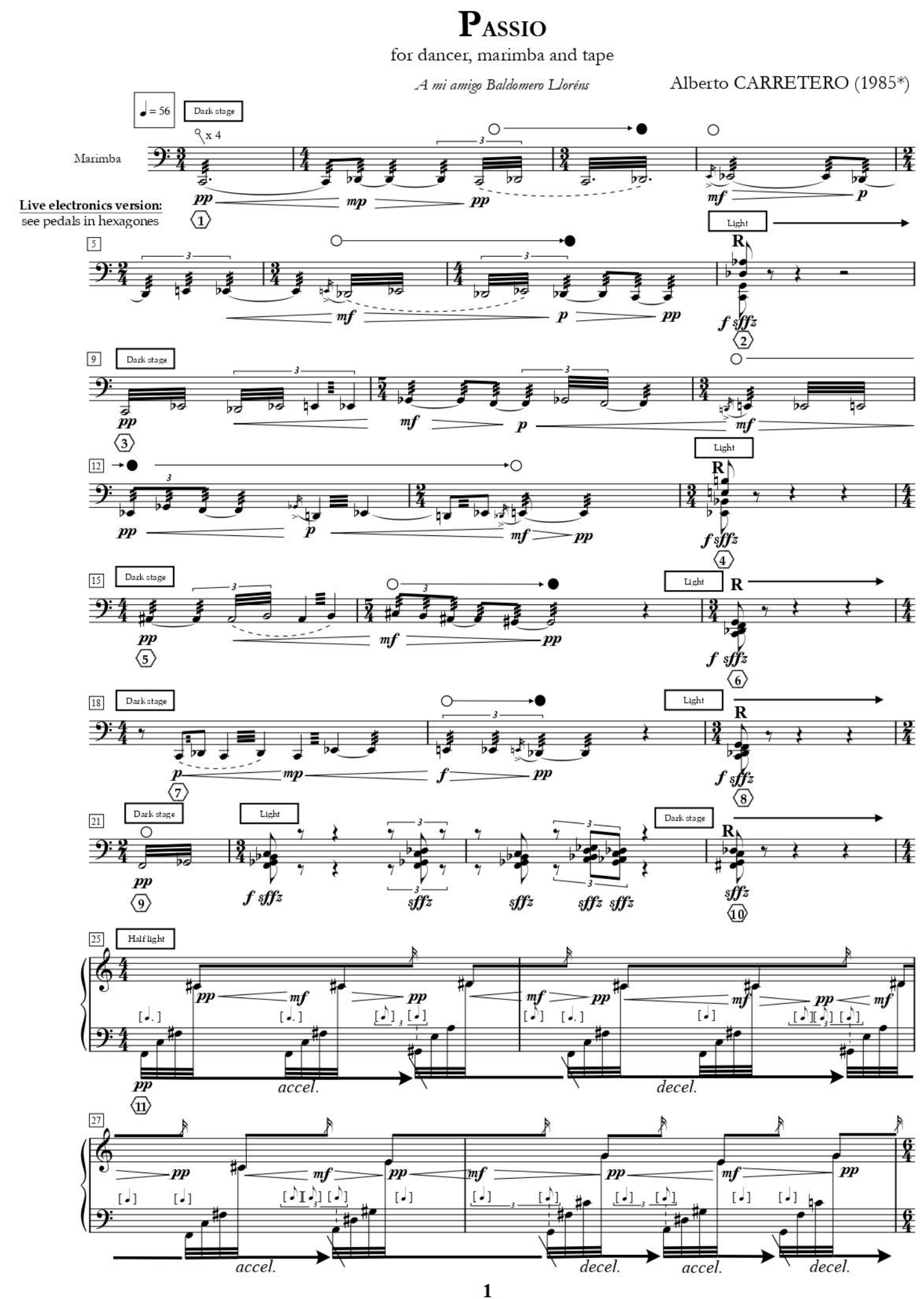
Enclaves. Revista de Literatura, Música y Artes Escénicas, n. ${ }^{\circ}$ 1, 2021, pp. 196-213. e-ISSN 2792-7350

Alberto Carretero Aguado, «PASSIO. Composición musical para marimba, danza, captación de movimiento y electrónica en vivo producida en IRCAMCentre Pompidou, París», https://dx.doi.org/10.12795/enclaves.2021.i01.13

\section{9:nclaves}

Revista de Literatura, Música y Artes Escénicas

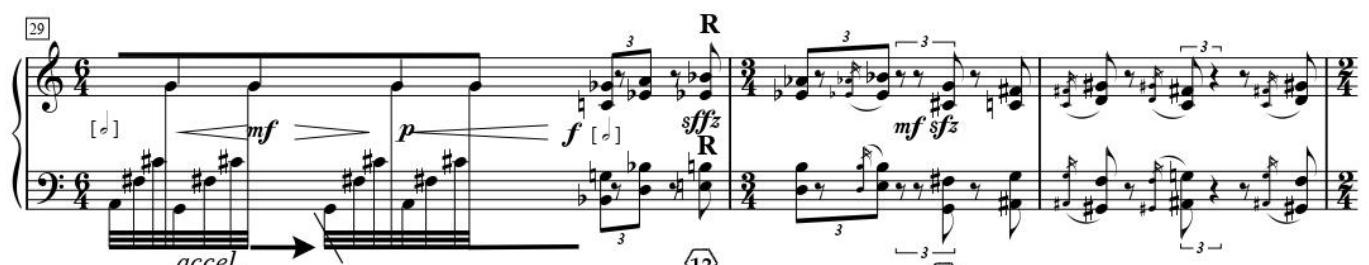

(12)

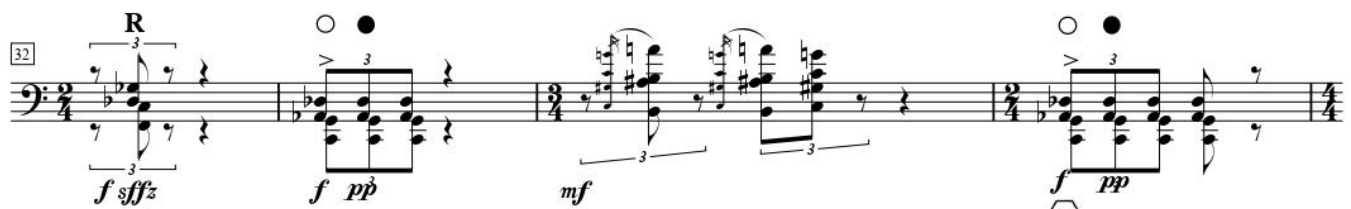

(14)

(15)

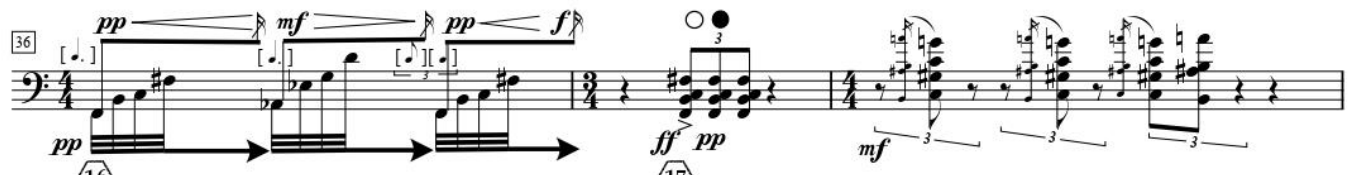
(16)

(17)

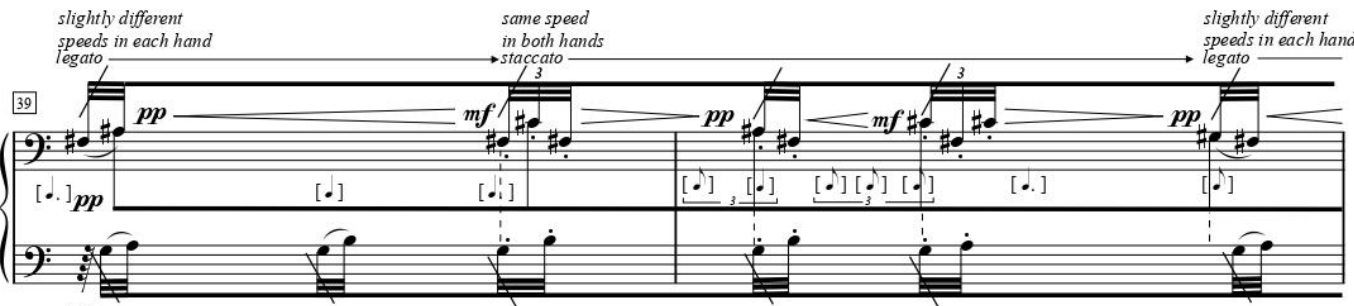

(18)
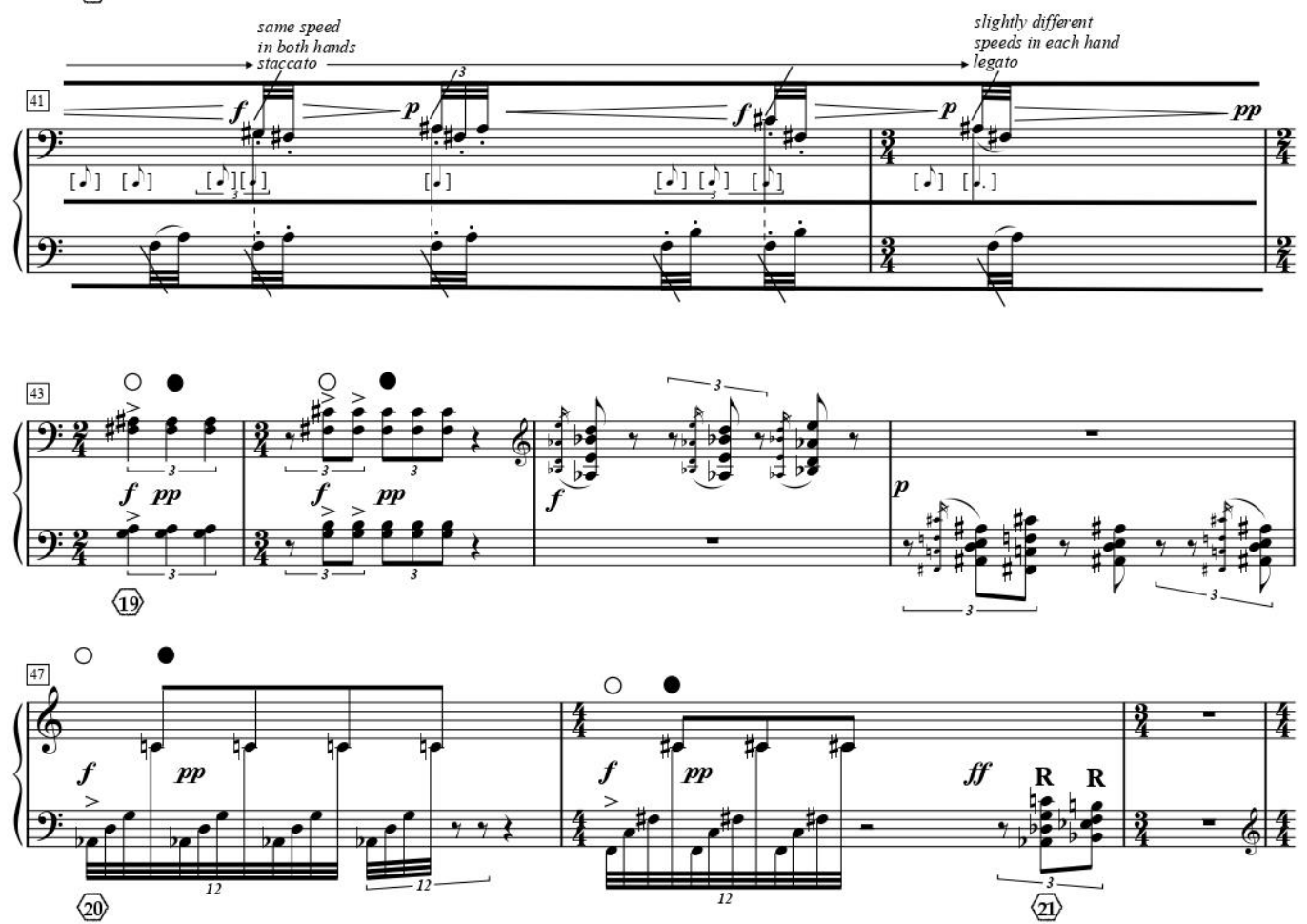
Enclaves. Revista de Literatura, Música y Artes Escénicas, n. ${ }^{\circ}$ 1, 2021, pp. 196-213. e-ISSN 2792-7350

Alberto Carretero Aguado, «PASSIO. Composición musical para marimba, danza, captación de movimiento y electrónica en vivo producida en IRCAMCentre Pompidou, París», https://dx.doi.org/10.12795/enclaves.2021.i01.13

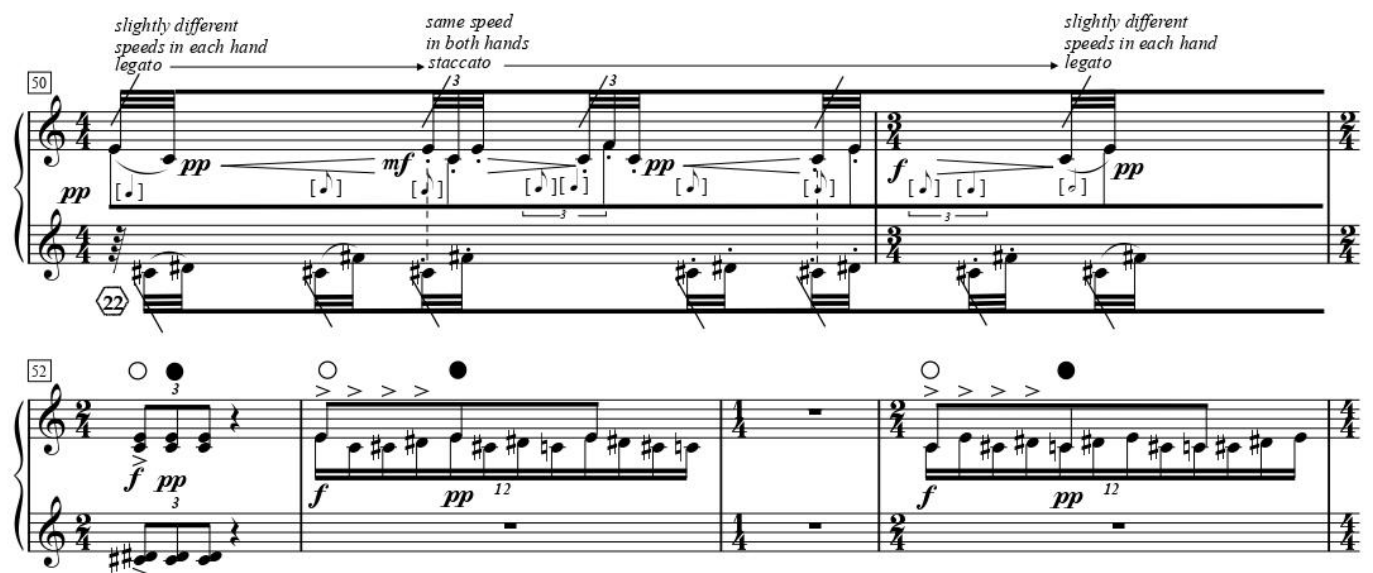

23 speed
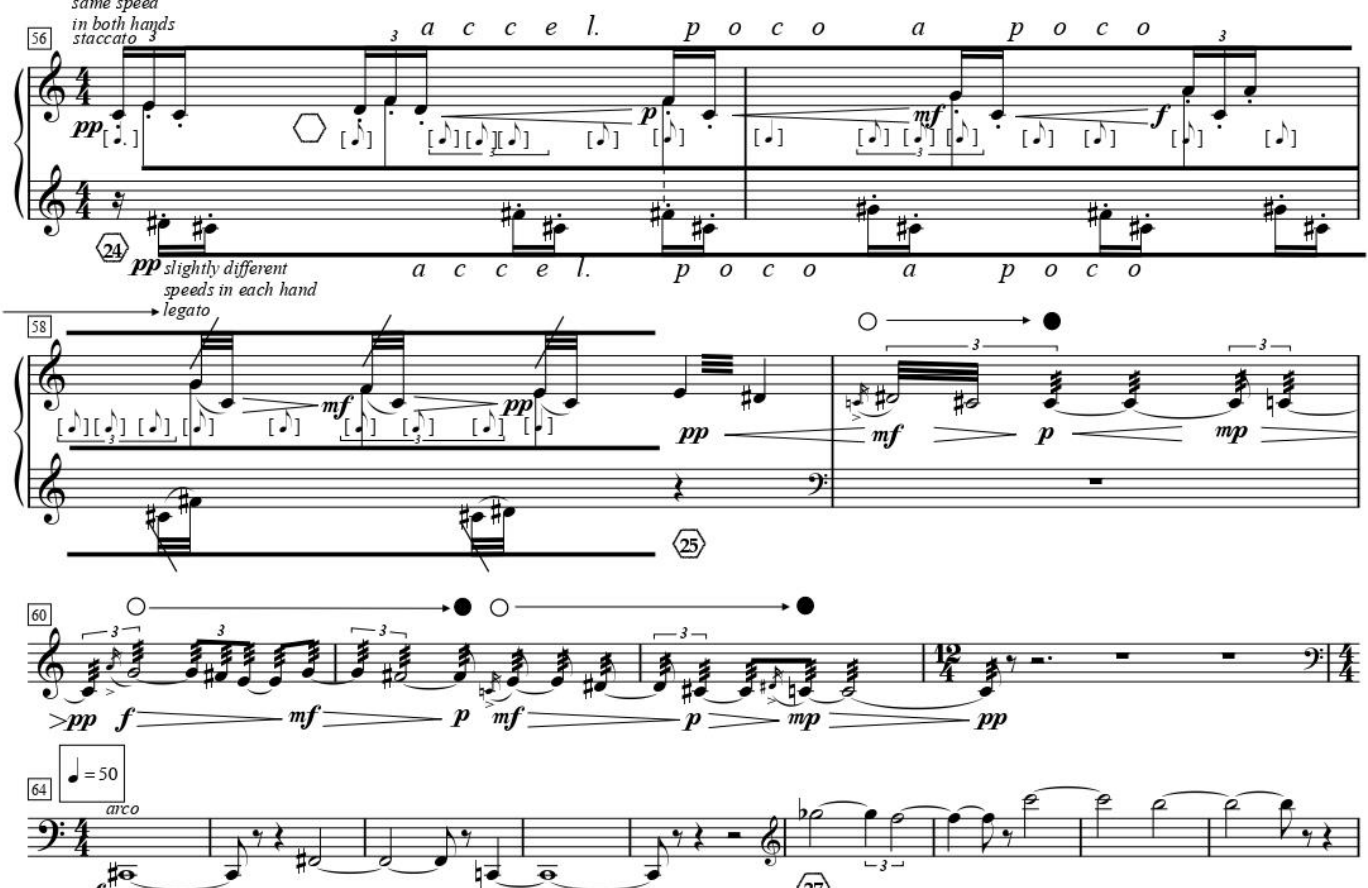

26.

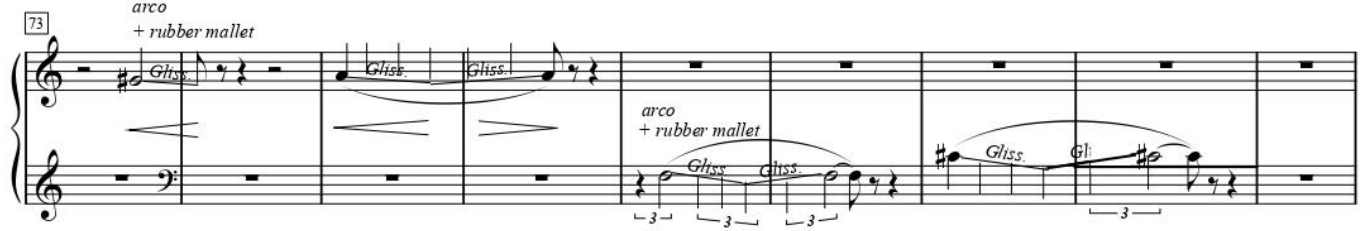

(28)

$\overline{(29)}$

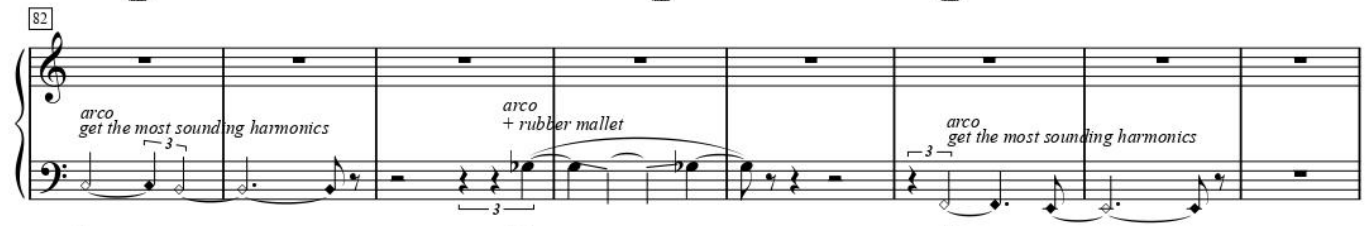

(31)

(32)

33 
Enclaves. Revista de Literatura, Música y Artes Escénicas, n. ${ }^{\circ}$ 1, 2021, pp. 196-213. e-ISSN 2792-7350

Alberto Carretero Aguado, «PASSIO. Composición musical para marimba, danza, captación de movimiento y electrónica en vivo producida en IRCAMCentre Pompidou, París», https://dx.doi.org/10.12795/enclaves.2021.i01.13

\section{9:nclaves}

Revista de Literatura, Música y Artes Escénicas

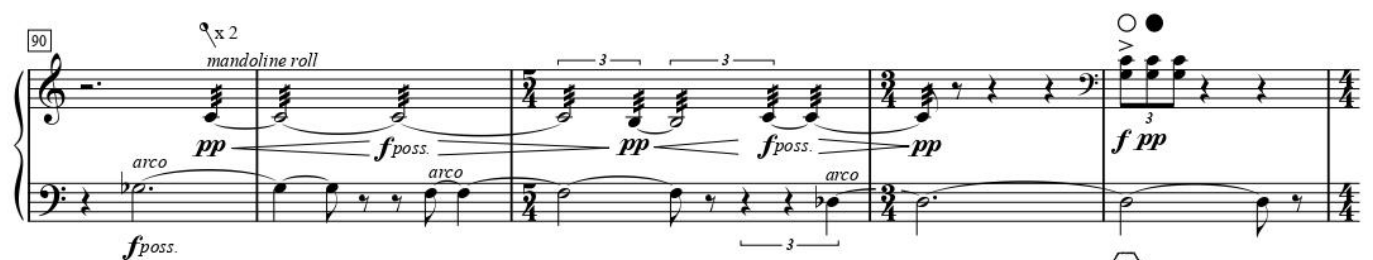

(34)

(35)
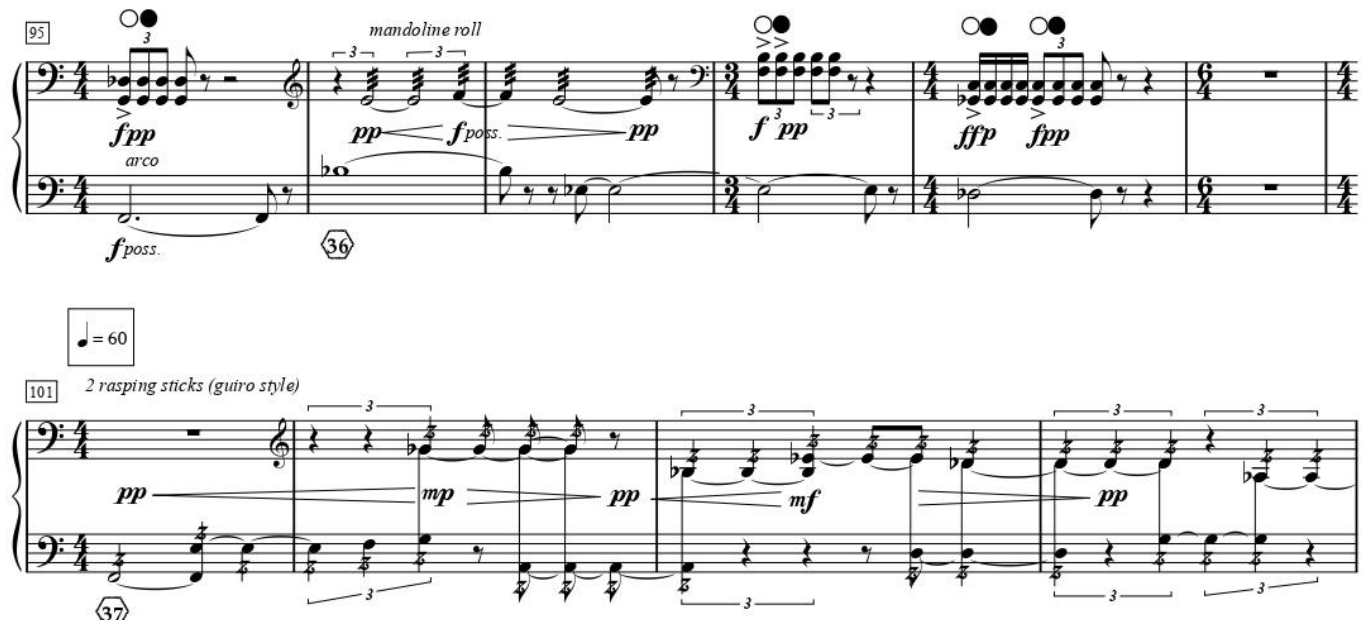

(37)
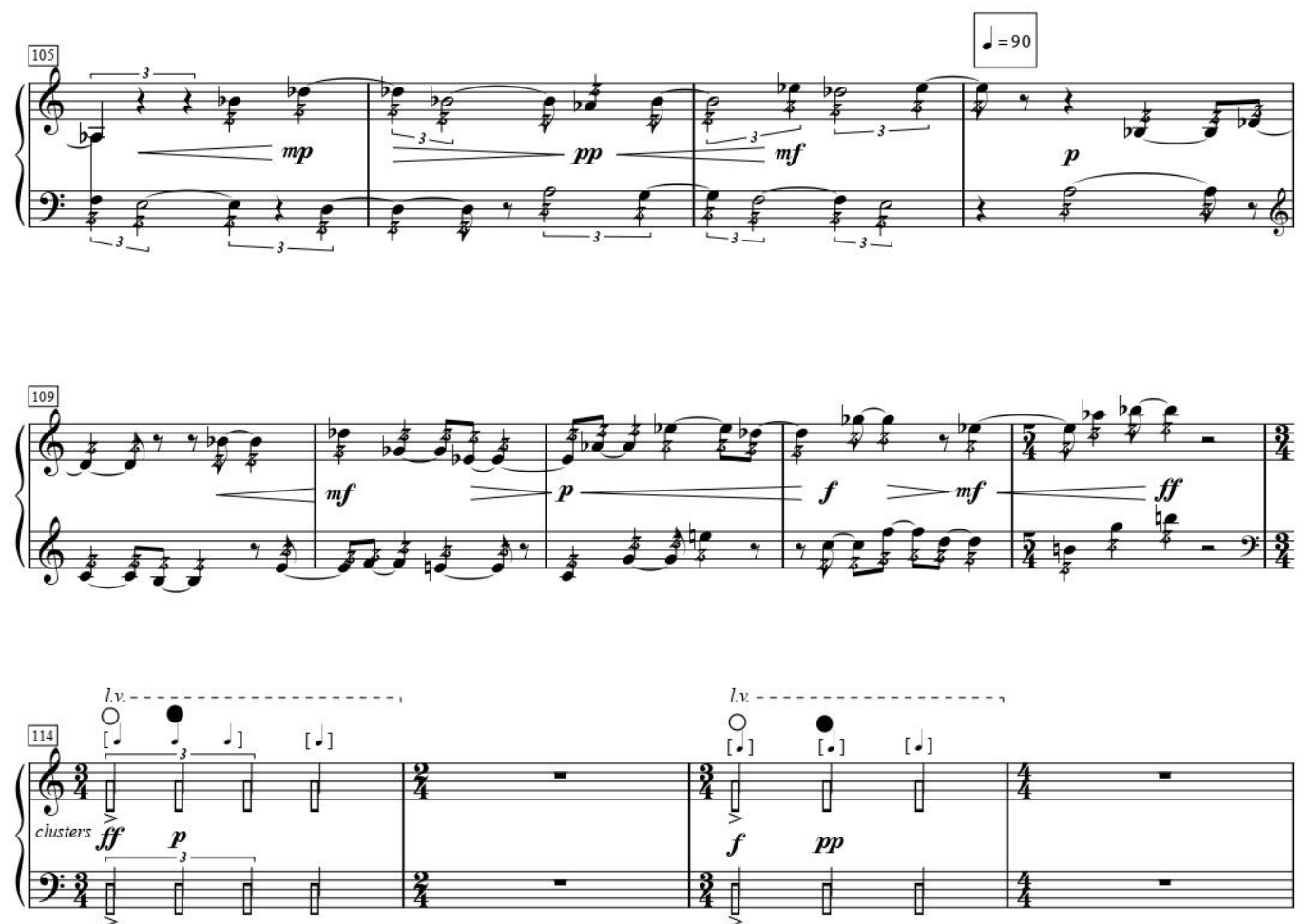

38 
Enclaves. Revista de Literatura, Música y Artes Escénicas, n. ${ }^{\circ}$ 1, 2021, pp. 196-213. e-ISSN 2792-7350

Alberto Carretero Aguado, «PASSIO. Composición musical para marimba, danza, captación de movimiento y electrónica en vivo producida en IRCAMCentre Pompidou, París», https://dx.doi.org/10.12795/enclaves.2021.i01.13
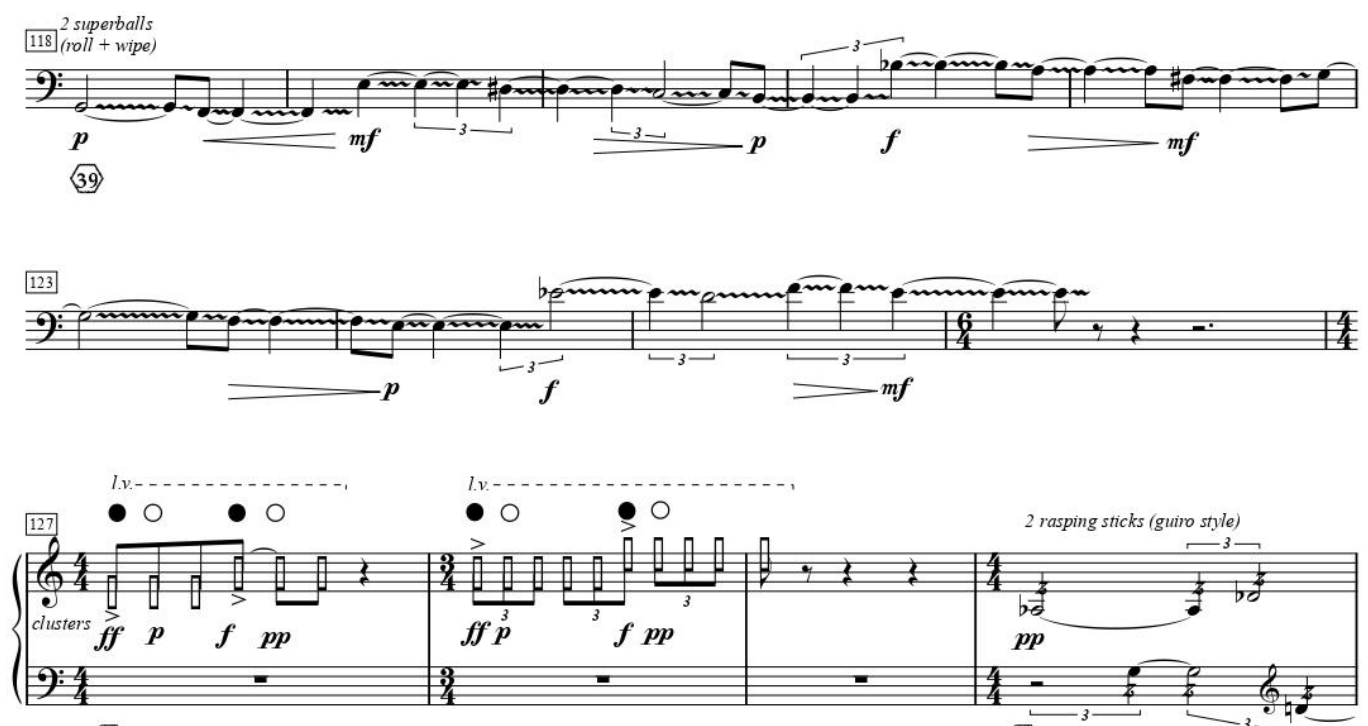

(40)

(411)
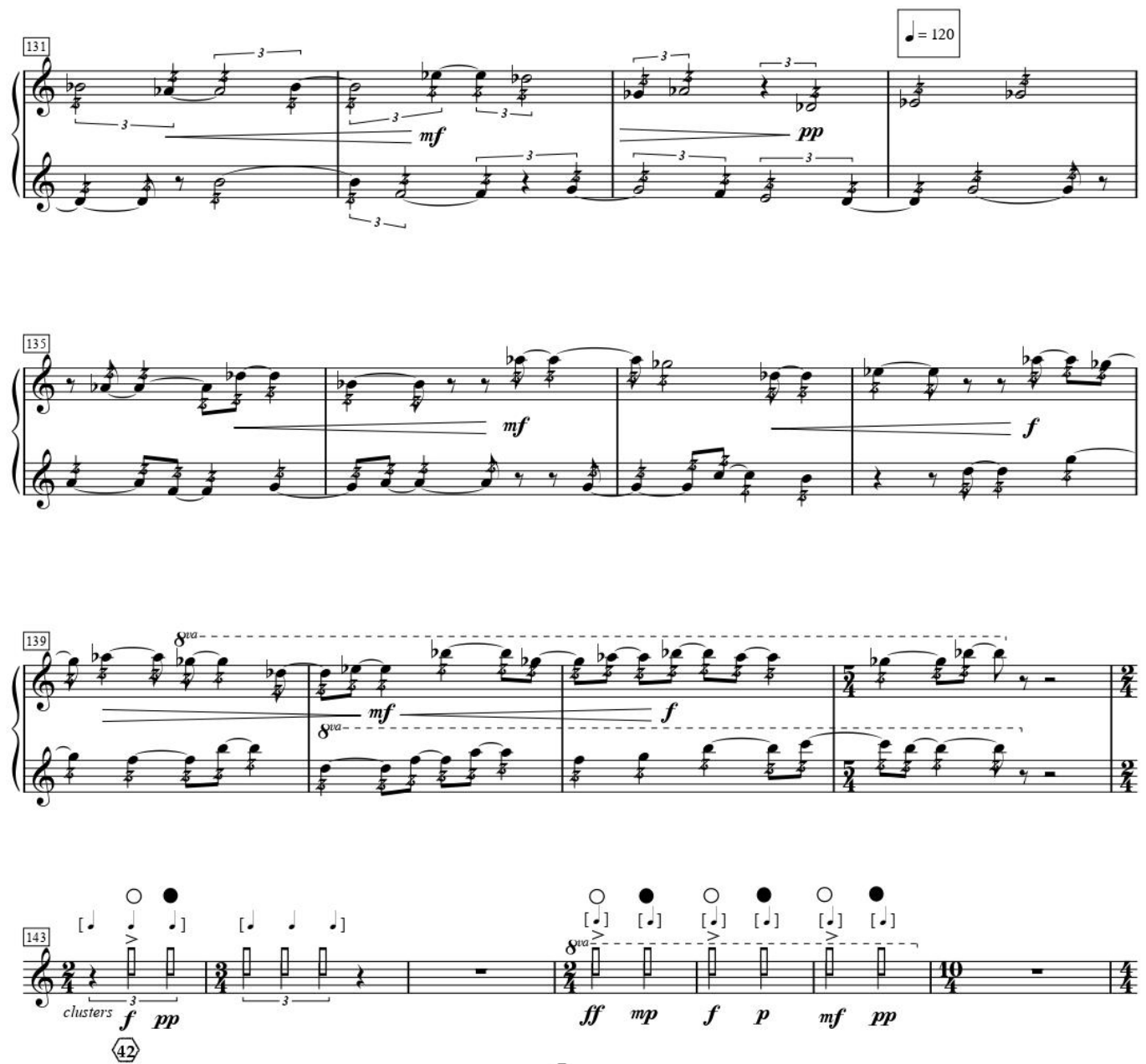
Enclaves. Revista de Literatura, Música y Artes Escénicas, n. ${ }^{\circ}$ 1, 2021, pp. 196-213. e-ISSN 2792-7350

Alberto Carretero Aguado, «PASSIO. Composición musical para marimba, danza, captación de movimiento y electrónica en vivo producida en IRCAMCentre Pompidou, París», https://dx.doi.org/10.12795/enclaves.2021.i01.13
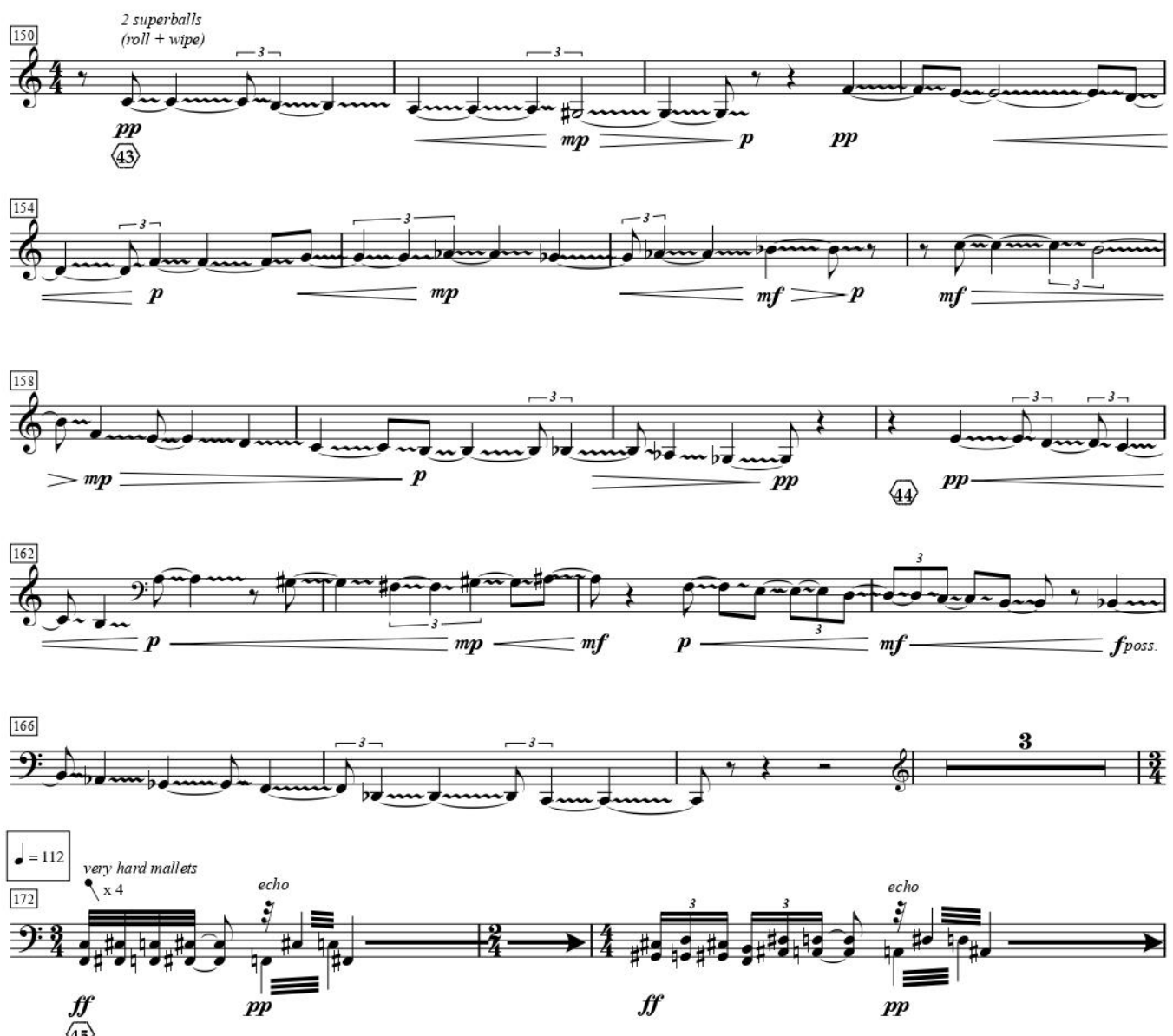
175
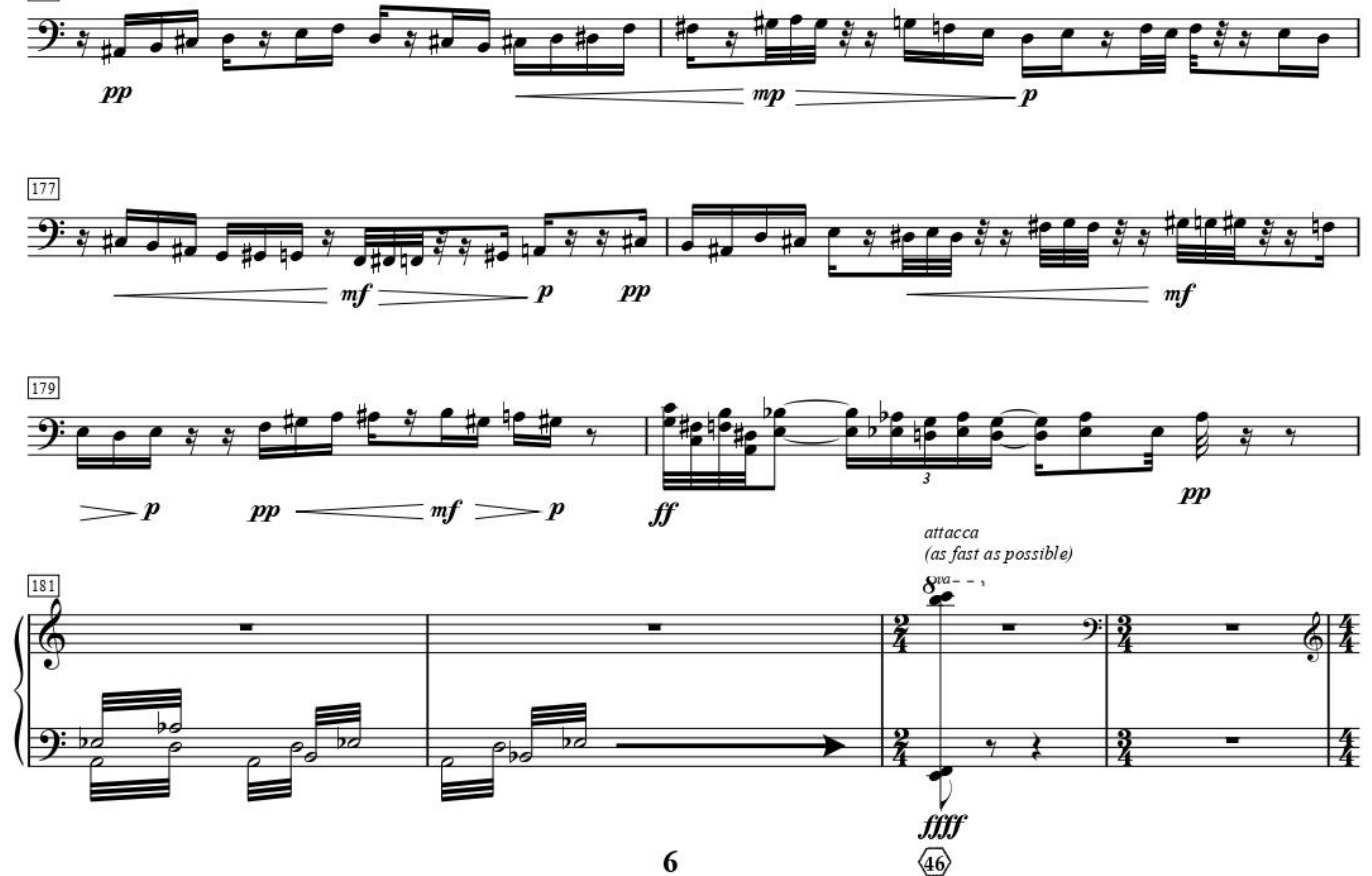
Enclaves. Revista de Literatura, Música y Artes Escénicas, n. ${ }^{\circ}$ 1, 2021, pp. 196-213. e-ISSN 2792-7350

Alberto Carretero Aguado, «PASSIO. Composición musical para marimba, danza, captación de movimiento y electrónica en vivo producida en IRCAMCentre Pompidou, París», https://dx.doi.org/10.12795/enclaves.2021.i01.13 9:nclaves

Revista de Literatura, Música y Artes Escénicas

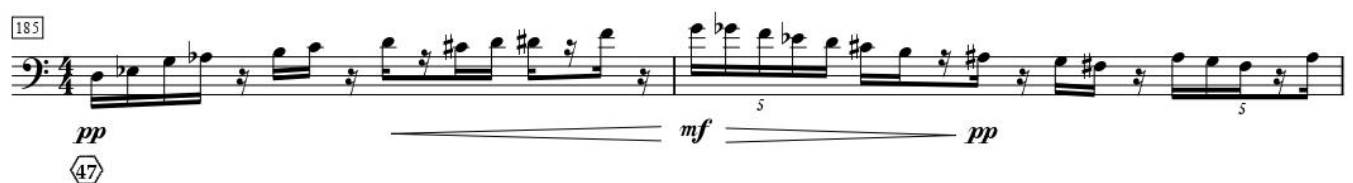

(47)
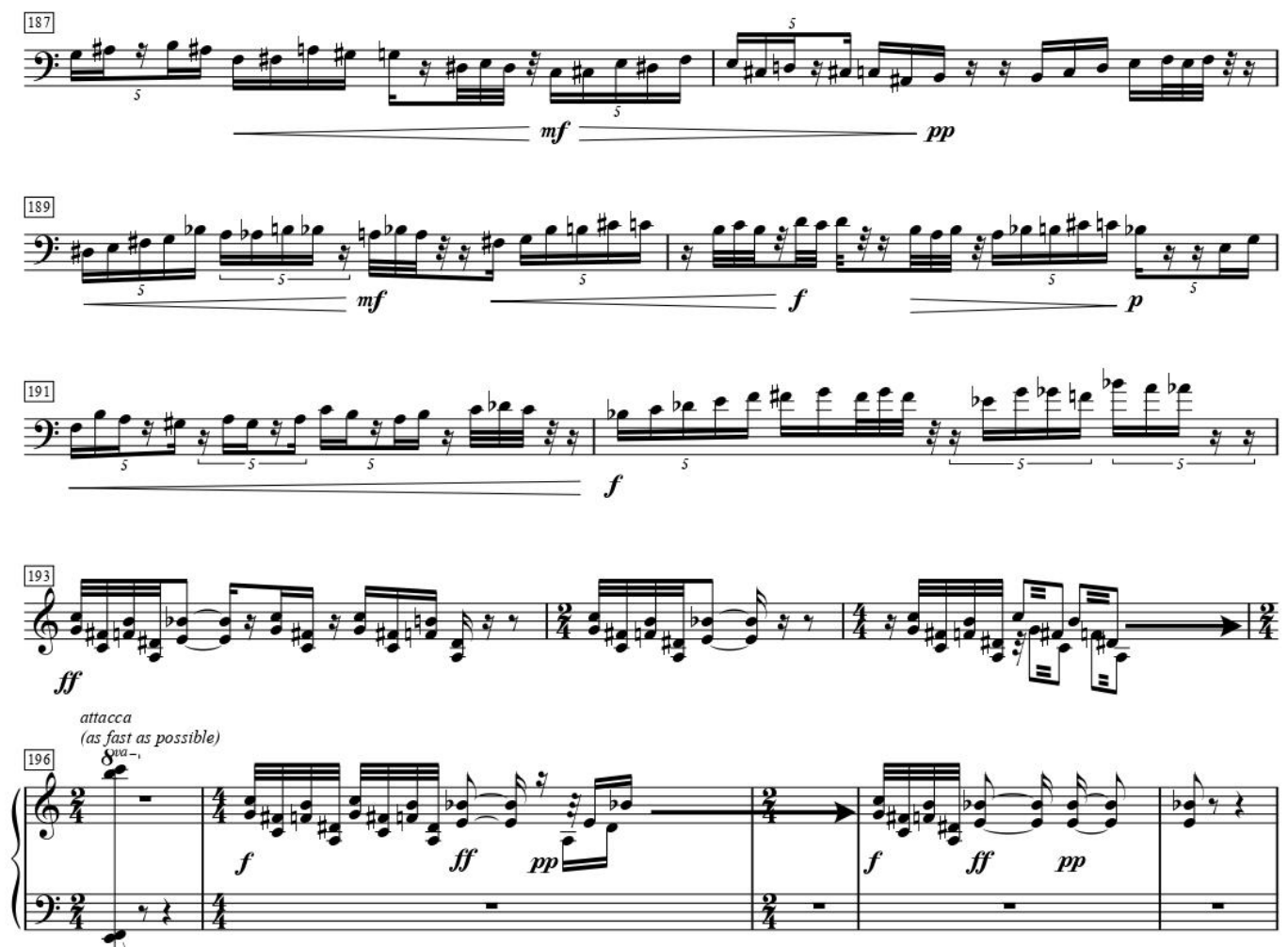

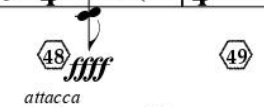
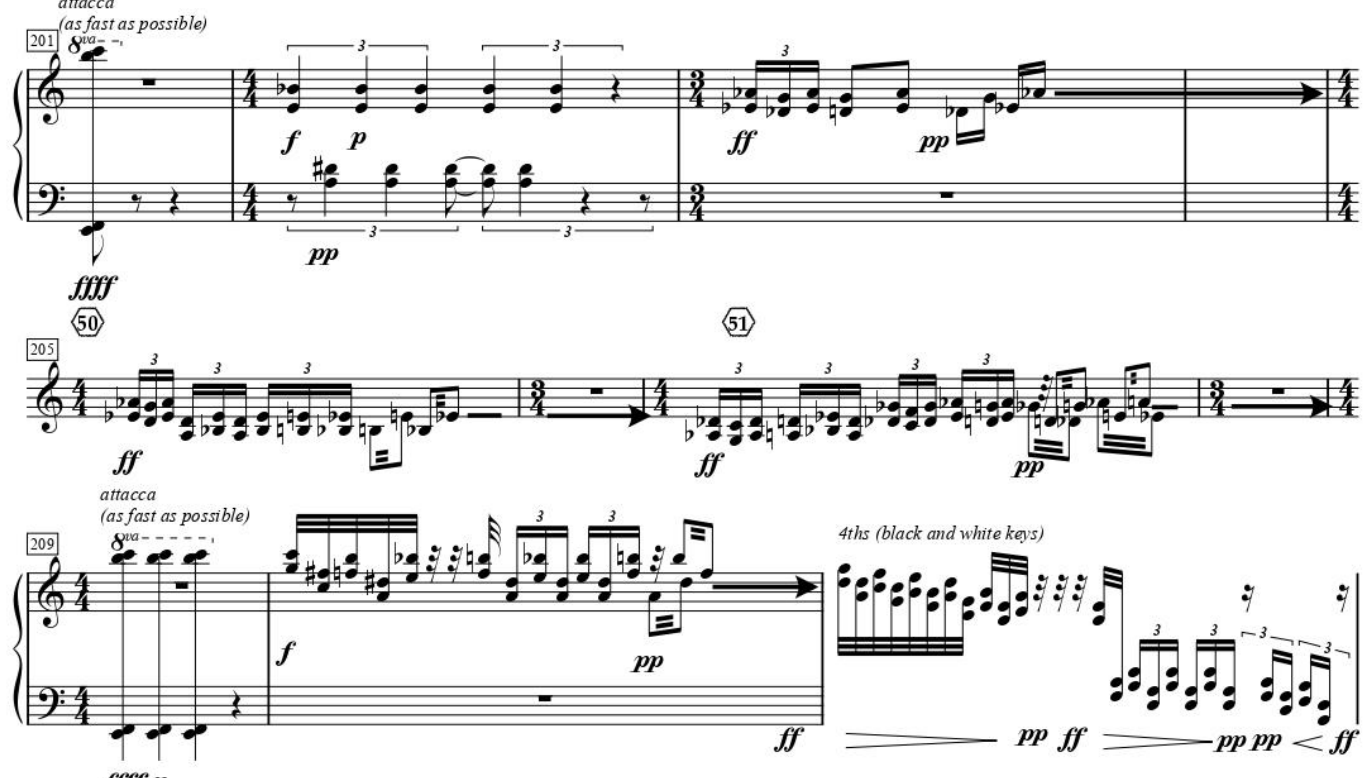

4ths (black and white keys)

ffff $p$

(52)

(53)

7

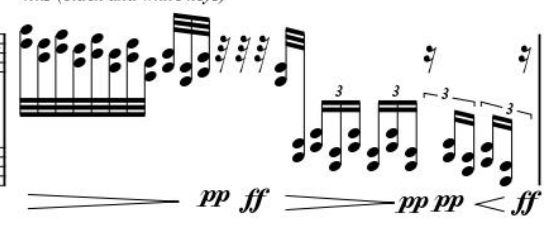


Enclaves. Revista de Literatura, Música y Artes Escénicas, n. ${ }^{\circ}$ 1, 2021, pp. 196-213. e-ISSN 2792-7350

Alberto Carretero Aguado, «PASSIO. Composición musical para marimba, danza, captación de movimiento y electrónica en vivo producida en IRCAMCentre Pompidou, París», https://dx.doi.org/10.12795/enclaves.2021.i01.13

\section{9:nclaves}

Revista de Literatura, Música y Artes Escénicas
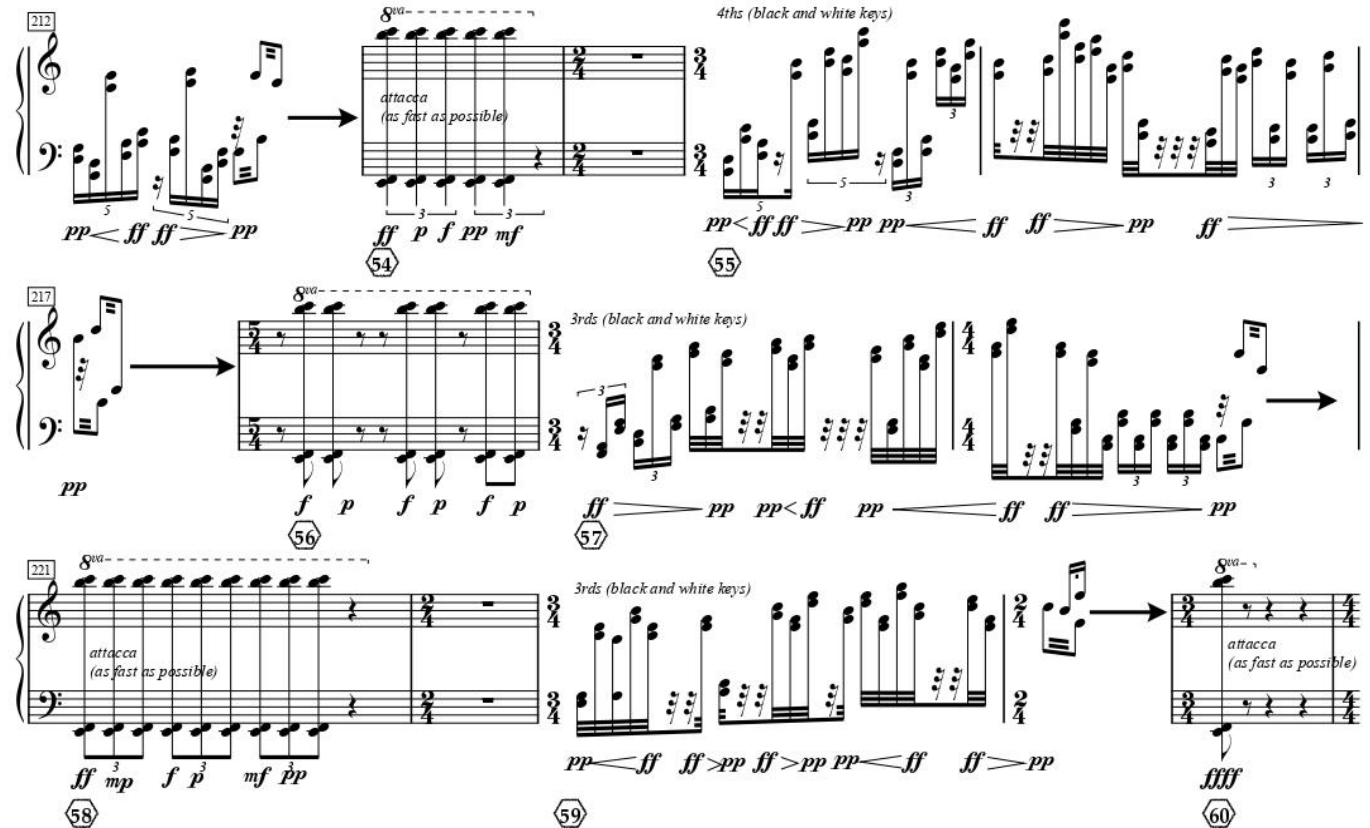

(58)

(59)

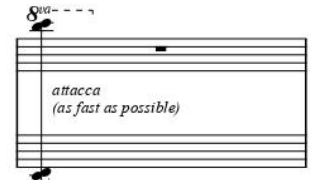

$\{: 4 \underbrace{226}$

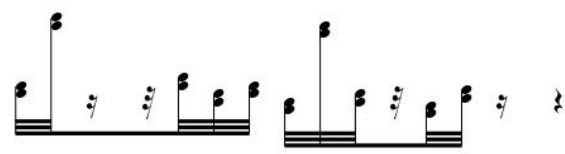

fisf

2 free improvisation (orientative written rhythm)
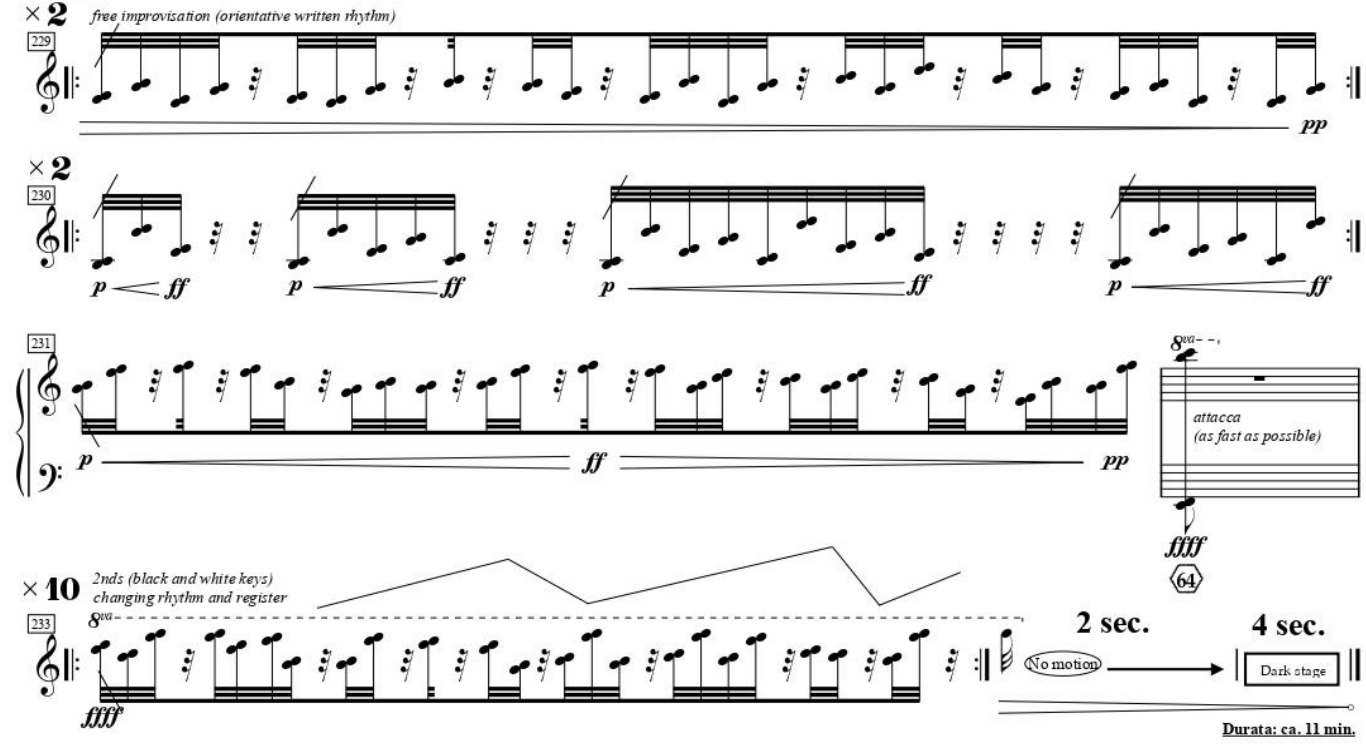

8

Imagen 6. 
Enclaves. Revista de Literatura, Música y Artes Escénicas, n. ${ }^{\circ}$ 1, 2021, pp. 196-213. e-ISSN 2792-7350

Alberto Carretero Aguado, «PASSIO. Composición musical para marimba, danza, captación de movimiento y electrónica en vivo producida en IRCAM-

Centre Pompidou, París», https://dx.doi.org/10.12795/enclaves.2021.i01.13

\section{Agradecimientos}

Gracias a Aurélie Berland, Linda Edsjö, Christine Gérard, Benoit Meudic, así como al equipo técnico y artístico del IRCAM-Centre Pompidou.

\section{Referencias}

Enlace a la referencia en la página web del IRCAM-Centre Pompidou:

https://medias.ircam.fr/embed/media/xbb9d6b_passio-for-dancer-marimba-and-live-elect

Enlace al catálogo del IRCAM-Centre Pompidou (página 156):

https://www.dropbox.com/s/e55y1gnt0i2n11s/ra-2013.pdf?dl=0

Enlace a la grabación en vídeo del estreno:

https://www.youtube.com/watch?v=JGbJLAP2ii4

Enlace a la grabación en audio de la música:

https://soundcloud.com/albertocarretero/passiocarretero

Enlace al programa del concierto:

https://www.dropbox.com/s/pa61lbxgx93rdbl/vivo_danse.pdf?dl=0 